\title{
Simulations of confined turbulent vortex flow
}

\author{
J.J. Derksen *,1 \\ Kramers Laboratorium voor Fysische Technologie, Delft University of Technology, \\ Prins Bernhardlaan 6, 2628 BW Delft, The Netherlands
}

Received 16 December 2003; received in revised form 26 April 2004; accepted 14 June 2004

Available online 16 September 2004

\begin{abstract}
Large-eddy simulations (LES) of the turbulent flow in a swirl tube with a tangential inlet have been performed. The geometry, and flow conditions were chosen according to an experimental study by [Escudier MP, Bornstein J, Zehnder N. Observations and LDA measurements of confined turbulent vortex flow. J Fluid Mech 1980;98:49-63]. Lattice-Boltzmann discretization was used to numerically solve the Navier-Stokes equations in the incompressible limit. Effects of spatial resolution and choices in subgridscale modeling were explicitly investigated with the experimental data set as the testing ground. Experimentally observed flow features, such as vortex breakdown and laminarization of the vortex core were well represented by the LES. The simulations confirmed the experimental observations that the average velocity profiles in the entire vortex tube are extremely sensitivity to the exit pipe diameter. For the narrowest exit pipe considered in the simulations, very high average velocity gradients are encountered. In this situation, the LES shows the most pronounced effects of spatial resolution and subgrid-scale modeling.
\end{abstract}

(C) 2004 Elsevier Ltd. All rights reserved.

\footnotetext{
* Fax: +1 6092580211 .

E-mail addresses: jderksen@princeton.edu, jos@klft.tn.tudelft.nl

${ }^{1}$ Presently on sabbatical leave at: Department of Chemical Engineering, Princeton University, The Engineering Quadrangle, Princeton, NJ 08544, USA.
} 


\section{Introduction}

Swirling flows have many technical applications. Combustion (with swirl for flame stabilization), turbo-machinery, and cyclone separators are a few examples. Experiments demonstrate that the swirl component has large-scale, and often counterintuitive effects on the flow field [1-3]. To mention a few phenomena: quasi-periodic oscillations (vortex core precession), subcritical flow (i.e. an influence of the downstream conditions on the entire flow), and vortex breakdown. Theoretical work on swirling flow [4,5] is often based on the Euler equations, leaving out turbulence. Industrial flows are, however, in many cases turbulent due to the large scale of the equipment, and high throughput. Predictive methods on swirling flow that incorporate turbulence are of great practical value, because in general turbulence strongly influences the performance of a process. In this paper, we explore to what extent large-eddy simulations (LES) based on the Smagorinsky subgrid-scale model, and two variants of it can be applied to describe turbulent, strongly swirling flow.

The research presented in this article originates from earlier work comprising LES of the flow in a reverse-flow cyclone for separating solid particles from a gas stream [6,7]. The motivation to use LES for cyclonic flows is twofold. In the first place, compared to RANS based simulations, particle transport (the foremost goal for cyclone modeling) can be incorporated much more realistically in a LES. Especially in the case of heavy particles in a gas stream, the resolved time-scales of the flow field can be made comparable to the relaxation time of the particles. This way, the fluctuations that are influential to particle motion are fully resolved, and particle trajectory calculations do not need further modeling [7]. In the second place, swirling flows often exhibit coherent, quasi-periodic fluctuations (e.g. the precessing vortex core, PVC [1,6-9]) that cannot be clearly separated from turbulent fluctuations in the frequency domain. The absence of a spectral gap between coherent and turbulent fluctuations makes LES a favorable approach compared to transient RANS. The outstanding challenges for RANS simulations of swirling flows were recently addressed by Jakirlic et al. [10].

In order to study the performance of our simulation procedure applied to strongly swirling flow in more detail, and to make use of an extensive body of experimental evidence on swirling flow systems it was decided to select a geometry with great similarity to a reverse-flow cyclone: the vortex tube geometry defined in the experimental study by Escudier et al. [11], see Fig. 1. Just as the reverseflow cyclone, it has a tangential inlet which can be directly incorporated in the simulation procedure, thereby avoiding the need to separately generate swirl-inflow conditions, such as done by Pierce and Moin [12] in the context of their LES. In contrast to the reverse flow generated in the cyclone, the vortex tube facility generates a unidirectional flow. By means of flow visualization and extensive LDA experiments, Escudier and co-workers showed the strong impact of an exit pipe contraction on the entire flow field. Furthermore, vortex breakdown was observed inside, or slightly upstream of the exit pipe. A third important observation when visualizing the flow was the clear distinction between the vortex core region where radial mixing is strongly suppressed, and the outer region that shows turbulent vortical structures (Taylor-Görtler vortices $[2,13]$ ). The large-eddy simulation of the vortex tube flow with a full representation of the geometry and operating conditions as used in the experiments is the subject of the study described in the present article.

The impact of modeling assumptions on the quality of the flow field predictions has been investigated by applying three spatial resolutions with a ratio of the linear size of the grid-spacing of 1:1.39:2.24, and by applying three different subgrid-scale (SGS) models, viz. the standard Smag- 


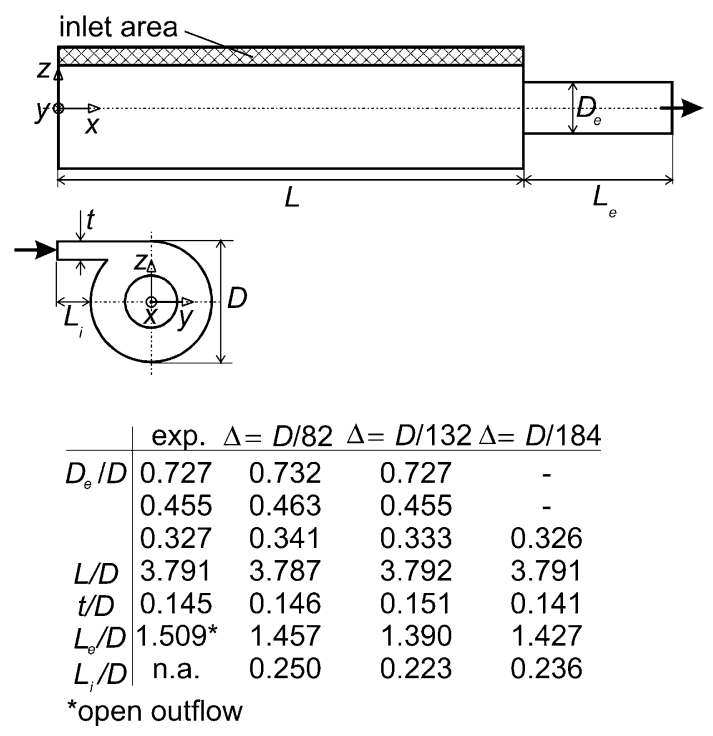

Fig. 1. Flow geometry as defined in [11], along with a coordinate system, and the dimensions in the experiment and in the simulations. Three exit pipe diameters have been considered $\left(D_{\mathrm{e}}=0.73 D, 0.46 D\right.$, and $\left.0.33 D\right)$, and three numerical grids (grid spacing $\Delta=D / 82, D / 132$, and $D / 184$ ).

orinsky model [14] with $c_{\mathrm{s}}=0.1$; the adaptation of the Smagorinsky model for low mesh-Reynolds-numbers due to Voke [15]; and a mixed-scale model due to Sagaut et al. [16]. All three models have been supplemented with Van Driest wall-damping functions [17] for bringing the eddy-viscosity to zero at solid walls.

The basis of our numerical procedure is a lattice-Boltzmann dicretization of the Navier-Stokes equations. Lattice-Boltzmann discretization [18] allows for second-order accurate flow simulations (in space and time) in arbitrarily shaped flow domains. Due to its computational efficiency (in terms of the number of operations per time step, and lattice-site), and locality ;of operations, highly resolved flow simulations are possible. The locality facilitates fully scalable simulations on parallel computer platforms. Incorporation of eddy-viscosity SGS models within lattice-Boltzmann discretization is straightforward, as will be demonstrated below.

This article is organized in the following manner: first the flow system is defined, including the boundary conditions that have been imposed in the simulations. Consecutively the simulation procedure, and modeling assumptions (i.e. the three SGS models that have been considered) will be described. Also a few remarks on the computational effort will be made. In presenting the results, the emphasis will be on confronting the simulations with experimental data, especially with respect to the influence of the spatial resolution and SGS models on the quality of the flow field predictions.

\section{Flow system}

The flow system, as introduced in [11] is given in Fig. 1. In this figure, a coordinate system, and the nomenclature with respect to the geometrical dimensions $\left(D, D_{\mathrm{e}}, L, L_{\mathrm{e}}\right.$, and $\left.t\right)$ have been 
defined. In [11], the definition of the Reynolds number was based on the conditions at the inlet: $R e=U_{\text {in }} t / v$, with $U_{\text {in }}$ the (superficial) inlet velocity $\left(U_{\text {in }}=Q / L t\right.$, with $Q$ the volumetric flow rate), and $v$ the fluid's kinematic viscosity. It should be noted that this Reynolds number definition is somewhat a-typical for the flow system under consideration, since the inlet conditions are not representative for the flow in the swirl tube and exit pipe. For instance, the Reynolds number based on the exit pipe diameter, and bulk velocity in the exit pipe is 5-25 times (dependent on $D_{\mathrm{e}}$ ) higher than the inlet Reynolds number. However, in order to conform with the definitions in [11], we will use the inlet Reynolds number in the remainder of this article to characterize the flow. In [11], also a geometrical swirl parameter was defined: $\Omega=\pi D D_{\mathrm{e}} / L t$. It serves as a measure for the ratio of angular to axial momentum.

In the experimental study, the emphasis was on the influence of the diameter of the exit pipe on the flow in the swirl tube. Many ratios $D_{\mathrm{e}} / D$ were considered in the experiment. From these three exit pipe diameters were selected: $D_{\mathrm{e}} / D=0.73,0.46$, and 0.33 (as indicated in the table in Fig. 1). The corresponding swirl parameters are $\Omega=4.2,2.6$, and 1.9 respectively. The experimental data consist of radial profiles of the time-averaged axial and tangential velocity measured with LDA at six axial positions. For brevity, only three of the six will be used in this paper (viz. $x / D=0.15$, 2.15, and 3.61). For $D_{\mathrm{e}} / D=0.727$ the Reynolds number was $R e=4800$. For $D_{\mathrm{e}} / D=0.455$, and $D_{\mathrm{e}} / D=0.327$ the LDA experiments were done at $R e=1900$. In measuring the radial velocity profiles, Escudier and co-workers took care to traverse their LDA measuring volume through the vortex core center (defined as the position with zero transverse velocity), which in general does not coincide with the geometrical center of the flow facility [11]. Furthermore, in [11] (and also in [13]) flow visualization photographs were presented at various Reynolds numbers, and various $D_{\mathrm{e}} / D$ ratios.

In our numerical study, the Reynolds numbers corresponded to the values used in the experiments. Furthermore, the case with the largest exit pipe diameter was also simulated at $R e=1900$. The simulations employed a uniform, cubic lattice (which is inherent for most lattice-Boltzmann schemes). In order to have an integer number of lattice spacings $\Delta$ in the vortex chamber diameter $D$, and in the exit pipe diameter $D_{\mathrm{e}}$, the values for the $D_{\mathrm{e}} / D$ ratios slightly deviate from the experimental values (see the table in Fig. 1). The simulations were carried out at three spatial resolutions: $\Delta=D / 82, \Delta=D / 132$, and $\Delta=D / 184$.

At the solid walls, no-slip boundary conditions were imposed. At the inlet area, the velocity in the $y$-direction was fixed to a parabolic function of the $z$-coordinate, and a uniform function of the $x$-coordinate. This laminar profile will not match physical reality, especially for the $R e=4800$ case. However, in the virulent region where the inlet flow merges with the swirling fluid already present in the body of the swirl tube, the fluid quickly forgets the conditions in the inflow channel (see also [7]). This was confirmed by tests with a uniform inlet velocity profile: no differences in terms of the average velocity field in the swirl tube with simulations using parabolic inlet profiles could be observed. The $x$ - and $z$-component of the velocity were set to zero at the inlet. At the outlet, a zero-gradient boundary condition was imposed. Only in one case (with the largest $D_{\mathrm{e}} / D$ ratio, and at $R e=4600$ ) unrealistic behavior of the flow starting at the exit boundary, and propagating upstream was observed which was probably due to subcriticality [13] at the exit plane. Application of a convective exit boundary strongly reduced (but not fully solved) the problem. 


\section{Simulation procedure}

Lattice-Boltzmann discretization of the Navier-Stokes equations has been applied. The specific scheme we used has been described by Somers and Eggels [19,20]. It explicitly takes into account third and fourth-order terms in the derivation of the Navier-Stokes equation from the lattice-Boltzmann equation, and shows stable behavior for low viscosity. It is at least second-order accurate in space and time. This makes the scheme well suited for simulation of turbulent flows [21]. Since the viscosity, and the deformation rate are directly contained in the lattice-Boltzmann scheme, the incorporation of eddy-viscosity SGS models is straightforward.

To represent curved, no-slip walls in the uniform, cubic lattice, a forcing technique has been developed [22], based on a similar technique developed by Goldstein et al. in the framework of spectral methods [23], and by Balaras [24], and Verzicco et al. [25] for finite volume schemes (the latter authors call it the immersed boundary technique). In our forcing technique, the wall surface is represented by a large set of control points $\mathbf{r}_{j}$ with a nearest neighbor distance slightly less than the lattice spacing. There is no restriction on the position of these points in the flow domain; they do not need to coincide with lattice sites. Every time step (with index $n$ ), the residual velocity at the control points $\mathbf{v}_{j}^{(n)}$ is determined by second-order interpolation from the velocity values at the nearest lattice-points $\mathbf{u}_{k}^{(n)}$ :

$$
\mathbf{v}_{j}^{(n)}=\sum_{k} G_{k}\left(\mathbf{r}_{j}\right) \mathbf{u}_{k}^{(n)}
$$

where $G_{k}$ is the interpolation function. To impose zero velocity at the points $\mathbf{r}_{j}$, the fluid is locally forced with

$$
\mathbf{f}_{j}^{(n)}=\alpha \mathbf{f}_{j}^{(n-1)}+\gamma \mathbf{v}_{j}^{(n)}
$$

where $\alpha$ and $\gamma$ are two parameters that have been empirically chosen to be 0.98 , and 1.9 respectively. The empiricism lies in the fact that the higher the values, the more accurate the zero velocity is achieved. However, when the parameters exceed specific values (that among other things depend on the spacing of the control points) the method rapidly becomes unstable. So far, no clear theory exists to derive the optimal $\alpha$ and $\gamma$. The final step is to distribute the forces at the control points (from Eq. (2)) over the lattice-Boltzmann nodes. For this goal, the same coefficients have been used as were used for the interpolation (i.e. the $G_{k}$ function):

$$
\mathbf{f}_{k}^{(n)}=\sum_{j} G_{k}\left(\mathbf{r}_{j}\right) \mathbf{f}_{j}^{(n)}
$$

The above method has been used extensively within the lattice-Boltzmann framework for imposing no-slip conditions at static and moving interfaces (see e.g. [6,7,22,26,27]). Derksen and Van den Akker [22] and Ten Cate et al. [27] performed a detailed assessment of the method by comparing its results to experimental data (LDA, PIV). They achieved good results for noslip boundary conditions at the surface of an impeller rotating in a mixing tank (operated under turbulent conditions), and at the surface of a solid sphere sedimenting in a closed box respectively. 


\section{Subgrid-scale modeling}

In modeling the flow fields in the reverse-flow cyclones [6,7], the standard Smagorinsky SGS model [14] with $c_{\mathrm{s}}=0.1$ was used exclusively. One of the goals of the present study is to assess the performance of this and two other SGS models for strongly swirling flow: Voke's low mesh-Re-number model [15], and Sagaut et al.'s mixed-scale model [16].

The three models are all based on the eddy-viscosity concept: the SGS-stresses are related to the resolved deformation rate via an eddy viscosity. The standard Smagorinsky model reads

$$
v_{\mathrm{e}, \mathrm{Sm}}=c_{\mathrm{s}}^{2} \Delta^{2} \sqrt{2 \bar{s}_{i j} \bar{s}_{i j}}
$$

with $v_{\mathrm{e}, \mathrm{Sm}}$ the eddy-viscosity, and $\bar{s}_{i j}$ the resolved rate of strain tensor. The constant $c_{\mathrm{s}}$ was set to 0.1 throughout the paper.

The Voke model is specifically designed for flows with regions that have low mesh Reynolds numbers $r=\frac{\Delta^{2}\left(2 \bar{s}_{i j} \bar{s}_{i j}\right)^{1 / 2}}{v}$, where it reduces the eddy viscosity compared to the standard Smagorinsky model according to

$$
v_{\mathrm{e}, \mathrm{Vo}_{\mathrm{o}}}=v_{\mathrm{e}, \mathrm{Sm}}-\beta v\left[1-\exp \left(-\frac{v_{\mathrm{e}, \mathrm{Sm}}}{\beta v}\right)\right]
$$

This function, including the constant $\beta=2 / 9$ was based on fitting to eddy-viscosity estimates that are in accordance with the Pao dissipation spectrum [15]. It is anticipated that Voke's model improves predictions for strongly swirling flow: laminarization has been observed in the vortex core, with very large flow structures (compared to the structures in the outer region), see e.g. [11]. Since these large structures are well within the range that can be resolved by the grid, the eddy-viscosity should be brought to zero there, which in principle is taken care of by Eq. (5).

The mixed-scale model (MSM) as described by Sagaut et al. [16], is based on a double filtering approach: next to the low-pass filter directly supplied by the computational grid, a second lowpass filter is used to extract the turbulent kinetic energy of the test field: $q_{c}=\frac{1}{2}\left[\tilde{\bar{u}}_{i}-\bar{u}_{i}\right]^{2}$, with $\bar{u}_{i}$ the resolved velocity field (i.e. filtered with a cut-off wavenumber of $\pi / \Delta$ ), and $\tilde{\bar{u}}_{i}$ the resolved field filtered by the second filter that usually has a length-scale of $2 \Delta$ associated to it [16]. The eddyviscosity in the MSM is a mixture of the Smagorinsky eddy-viscosity, and the eddy-viscosity due to the turbulent kinetic energy (TKE) model:

$$
v_{\mathrm{e}, \mathrm{MSM}}=\sqrt{v_{\mathrm{e}, \mathrm{Sm}} v_{\mathrm{e}, \mathrm{TKE}}}
$$

with

$$
v_{\mathrm{e}, \mathrm{TKE}}=c \Delta \sqrt{q_{c}}
$$

The constant $c$ amounts to 0.01 [16].

In all cases, the eddy-viscosity was explicitly brought to zero in the vicinity of no-slip walls by Van Driest [17] damping functions:

$$
v_{\mathrm{e}, \mathrm{dmp}}=v_{\mathrm{e}, \mathrm{udmp}}\left(1-\exp \left(-\frac{y^{+}}{A^{+}}\right)\right)^{2}
$$

with $v_{\mathrm{e}, \mathrm{udmp}}$ the (undamped) eddy-viscosity from one of the three models discussed above, and $v_{\mathrm{e}, \mathrm{dmp}}$ the eddy-viscosity after applying wall-damping. The constant $A^{+}$amounted to 26 [17]. A 
uniform wall shear velocity $u^{*}=0.04 U_{\text {in }}$ was used for determining $y^{+}=\frac{y u^{*}}{v}(y$ is the distance to the wall). Our cyclone simulations [6] also applied a uniform $u^{*}$. The assumed value of $u^{*}$ was a postorioiri compared to the values calculated from the resulting average flow field. Assumed and calculated values were in fair agreement (a sensitivity study with respect to the imposed $u^{*}$ showed insignificant changes in the flow field in a $u^{*}$-range that was larger than the observed deviations between the imposed and the a postoriori calculated $u^{*}$ ).

\section{Overview of the simulated cases}

The large number of physical $\left(R e, D_{\mathrm{e}} / D\right)$ and numerical variables (grid-spacing, SGS model) prohibited a comprehensive set of simulations. The cases that we did study are summarized in Table 1. As can be seen, the focus is on the case with $D_{\mathrm{e}} / D=0.33$. The experiments show that a small $D_{\mathrm{e}} / D$ ratio leads to high tangential velocities throughout the geometry, and very sharply peaked axial velocity profiles. As a consequence, it is anticipated that the effects of spatial resolution, and (overly) dissipative SGS models are most pronounced for cases with a narrow exit pipe.

Tests showed that an averaging time of five or more integral time-scales $D / U_{\text {in }}$ was sufficient for statistically converged average velocity profiles. Higher order moments may require longer runs. To limit the computational effort, and since the validation is based on average velocities only, most simulations were run for five till ten integral time-scales. The simulations on the most course grid were run longer (for testing purposes); the one on the finest grid (Case \# 10) was run for only $2.8 \mathrm{D} / U_{\text {in. }}$ For the latter simulation some care in interpreting the velocity profiles should be taken.

The simulations were run in parallel on an in-house PC-cluster (Beowulf cluster). Because of the low communication load of the lattice-Boltzmann methodology, such low-cost PC clusters are well able to run the code efficiently (i.e. without much overhead). The cases with the intermediate grid $(D / \Delta=182)$ were mostly run on 6 cpu's in parallel. Total memory requirements for such cases are approximately $1.5 \mathrm{~Gb}$; runtimes for completion of one integral time-scale are of the order of 30 h (Pentium III, $700 \mathrm{MHz}$ PCs).

Table 1

Overview of numerical cases

\begin{tabular}{lllrlrl}
\hline Case \# & $D_{\mathrm{e}} / D$ & $R e$ & $D / \Delta$ & SGS model & $T U_{\text {in }} / D$ & Remarks \\
\hline 1 & 0.73 & 2100 & 82 & Standard Smagorinsky & 26.0 & \\
2 & 0.73 & 2100 & 132 & Standard Smagorinsky & 5.4 & Convective exit b.c. \\
3 & 0.73 & 4600 & 132 & Standard Smagorinsky & 7.3 & \\
4 & 0.45 & 2000 & 82 & Standard Smagorinsky & 10.0 & \\
5 & 0.45 & 2000 & 132 & Standard Smagorinsky & 9.3 & \\
6 & 0.33 & 2000 & 82 & Standard Smagorinsky & 12.8 & \\
7 & 0.33 & 1900 & 132 & Standard Smagorinsky & 11.2 & \\
8 & 0.33 & 1900 & 132 & Vokes model & 6.4 & \\
9 & 0.33 & 1900 & 132 & MSM & 6.4 & Short averaging time \\
\hline
\end{tabular}

In the last but one column, the averaging time $T$ has been indicated. 


\section{Results}

The first case that was simulated (Case \#1, see Table 1) was started from a zero velocity field. It took a long time before the swirl that was generated by the inlet boundary condition manifested itself in the entire geometry, and the flow became quasi-steady. For this reason, all subsequent simulations were started by taking a developed field. In case of a Reynolds number change, the viscosity was adapted; in case the SGS model was changed, the old model was simply switched off, and the new model was switched on. When the grid was refined, the coarser field was linearly interpolated to the new grid. When the exit pipe diameter was changed, a new geometry file (consisting of the forcing points, as explained in the Simulation Procedure section) was imported into the code. Subsequently, the forcing routine automatically imposed the new boundary conditions, and the flow field adapted itself accordingly.

As an example of the way the flow adapts itself to a new grid, the transition from Case \#7 to $\# 10$ (from $\Delta=D / 132$ to $\Delta=D / 184$ and further the same conditions) in terms of the $y$-velocity at the centerline is given in Fig. 2. It is difficult to distinguish a clear trend in the time-series, albeit that during the first integral time-scale $D / U_{\text {in }}$ after the refinement the velocity seems to develop towards a new quasi-steady state. In Fig. 2, the part of the time series that has been used to extract time averaged velocity data has been indicated as well.

\subsection{Flow visualization}

A laser sheet visualization image, reprinted from [11], shows the very sharp distinction between the clearly turbulent outer flow with large ring vortices (of the Taylor-Görtler type), and the much less turbulent vortex core (Fig. 3). The core is slightly bended, apparently due to the asymmetry

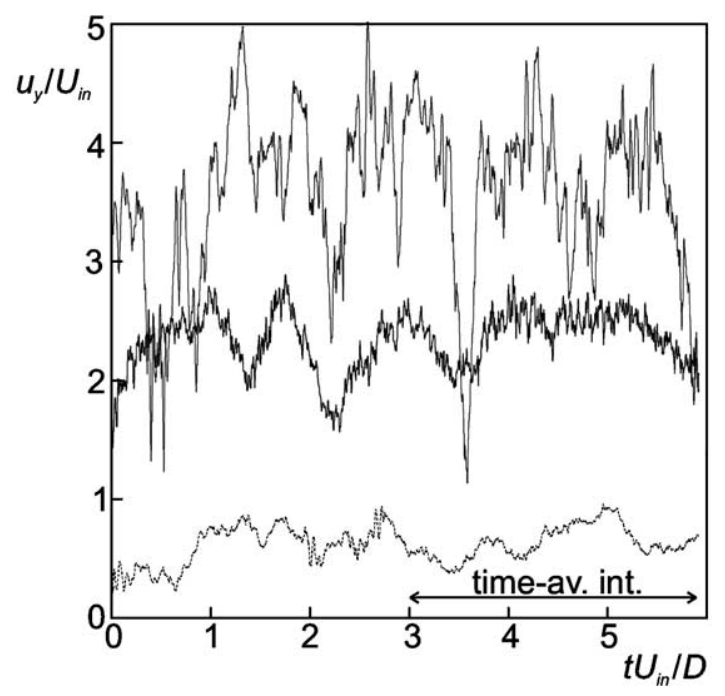

Fig. 2. Time series of the velocity in $y$-direction at the center of the vortex tube of the start-up of Case \#10 from an interpolated field retrieved from Case \#7. The axial position are $x / D=0.67$ (solid black), $x / D=1.83$ (dashed), and $x /$ $D=3.61$ (dotted). 


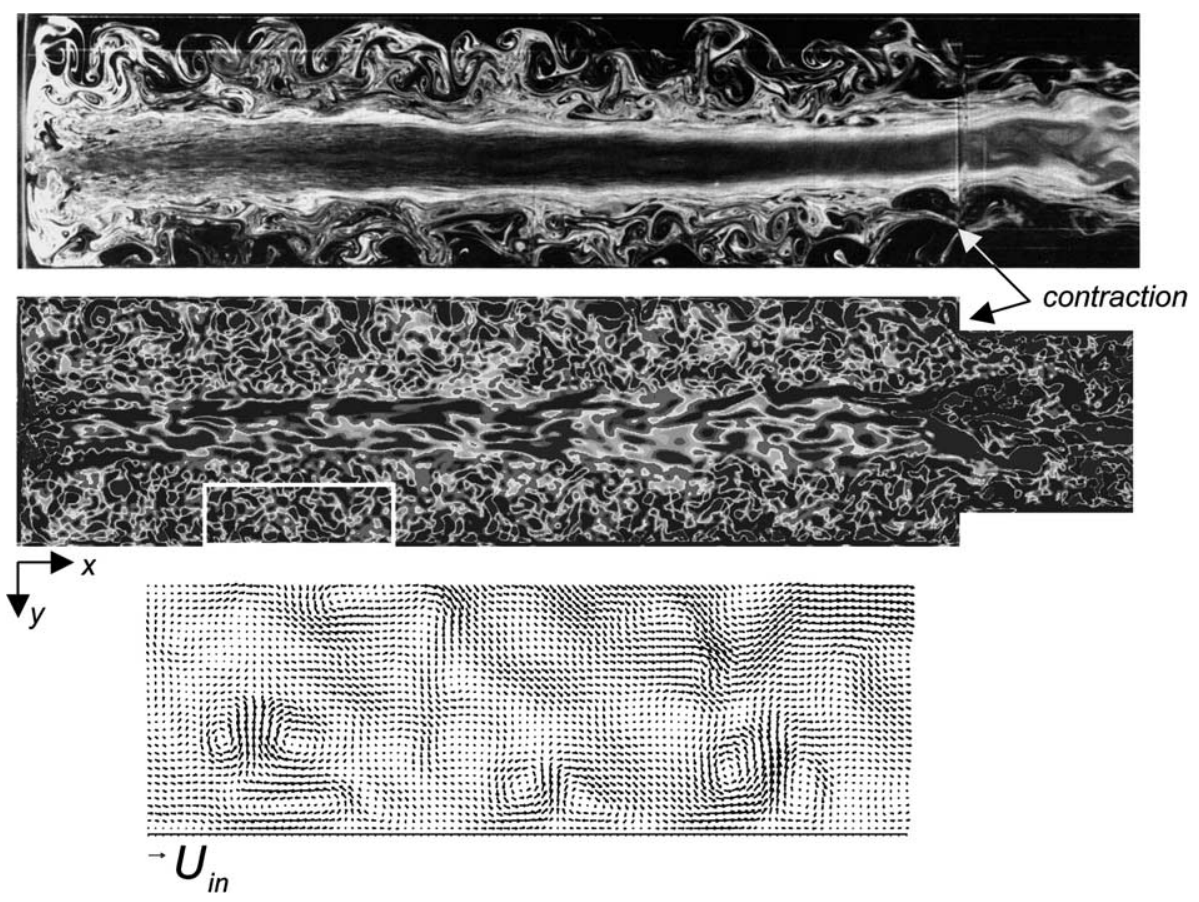

Fig. 3. Flow visualization of the entire vortex tube. Top: experiment (reprinted from [11]) at $R e=410, D_{\mathrm{e}} / D=0.58$. Center: LES snapshot at $R e=2100, D_{\mathrm{e}} / D=0.73$ (Case \#2) in terms of the vorticity. In both images, clearly the distinction between the laminar-like core, and the turbulent outer region can be observed. Bottom: the (instantaneous) velocity vectors in the part of the LES indicated with the white rectangle in the center figure.

induced by the tangential inlet. For qualitative comparison (the difference in Reynolds number between experiments and LES inhibits a more quantitative analysis), Fig. 3 shows the result of the LES (Case \#2) in terms of the vorticity, in the same plane as was illuminated in the experiment. As in the experiments, the vortex core can be clearly distinguished from the outer part of the flow: large-scale, coherent structures characterize the core; much smaller, erratic structures the outer flow. The much higher Reynolds number in the simulation is responsible for the smaller flow structures in the outer flow compared to the outer flow in the experiment. In the outer flow region, the lower part of the LES vorticity field shows slightly larger structures than the upper part. This is due to the closer proximity of the inlet channel to the lower part; the flow is still developing in the circumferential direction. The pairs of Taylor-Görtler vortices at the perimeter of the swirl tube with their strong radial inflow in between two vortices forming a pair have been highlighted in the vector plot, contained in Fig. 3.

Under certain conditions, the flow exhibits vortex breakdown (see Fig. 4). The occurrence and type of breakdown depends on the flow geometry (in this study the ratio $D_{\mathrm{e}} / D$ ), and the flow rate [28]. The experimental visualizations in Fig. 4 indicate a bubble type breakdown (Type 0 in the classification by Faler and Leibovich [28]) in the two geometrical cases shown. For $D_{\mathrm{e}} /$ $D=0.33$, the LES (Case \#7) has approximately the same Reynolds number as the experiment. It clearly shows the Type 0 breakdown, with a single, thin tail. The bubble is filled and emptied simultaneously at the rear part (see the vector plot in Fig. 4). Also note the laminar-turbulent 

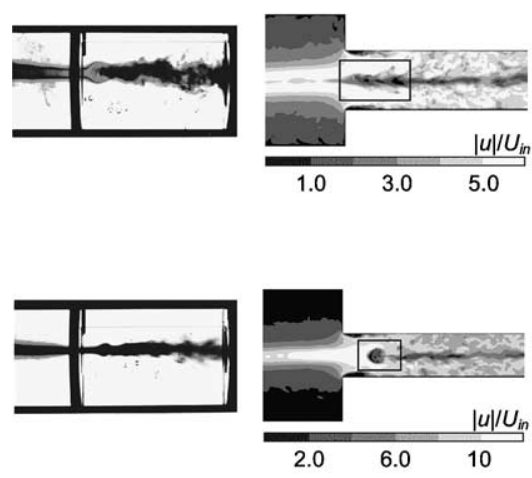
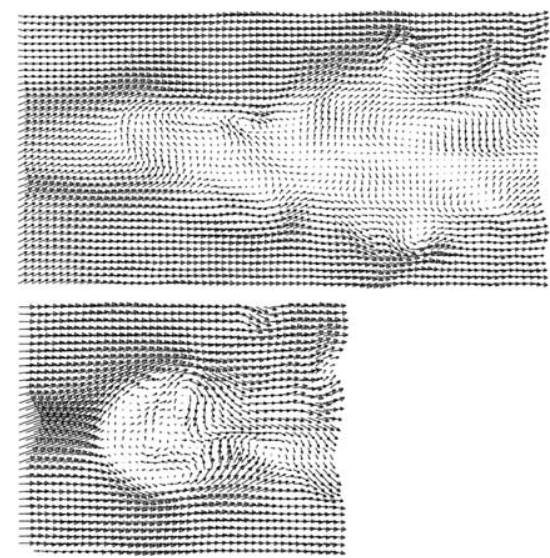

Fig. 4. Vortex breakdown for $D_{\mathrm{e}} / D=0.45$ (top), and $D_{\mathrm{e}} / D=0.33$ (bottom). Left: visualization as reported in [11] at $R e=650$ (top), and $R e=1600$ (bottom). Center: LES snapshot in terms of the contours of the absolute value of the velocity near the contraction at $R e=1900$, with $\Delta=D / 132$ (Cases \#5 and \#7). Right: the velocity vector field at the position as indicated with the rectangles in the center pictures.

transition over the breakdown bubble: upstream of the bubble the vortex core is laminar-like, whereas downstream of the bubble the flow is strongly turbulent over the entire cross-section of the exit tube. This experimentally observed feature is well represented in the LES. The dynamic behavior of the vortex breakdown has been visualized in Fig. 5, showing a time-sequence of velocity magnitude contours. The axial position of the breakdown bubble slightly fluctuates, and sometimes almost detaches from low-velocity region in the center of the exit pipe. In all instances shown in Fig. 5, the structure of the breakdown bubble in terms of velocity vectors is very similar to the vector plot shown in the bottom-right part of Fig. 4. For $D_{\mathrm{e}} / D=0.45$, there is less correspondence between experiment and LES. This may very well be due to the difference in Reynolds number in experiment and LES. Escudier et al. [11] experimentally observed an upstream migration of the breakdown when increasing the Reynolds number. In the LES at $D_{\mathrm{e}} / D=0.45$ (Case \#5), the breakdown appears to be of Type 1 (compared to Type 0 , Type 1 has a less regular shape and thicker tail, but still a stagnation point at the front side). Faler and Leibovich [28] indeed report Type 0 to Type 1 transitions when increasing the Reynolds number.

When looking at cross-sectional planes in the flow (Fig. 6), in general the vortex core in the swirl tube is a single, stable, coherent spike of vorticity. It is surrounded by a turbulent outer flow, as we already observed in Fig. 3. In the outer flow the wall boundary layers can be identified by their elevated vorticity levels. Also the way the boundary layer at the bottom wall of the inlet channel is swept into swirling flow can be clearly observed.

The laminar-turbulent transition of the flow at the vortex breakdown as witnessed in Figs. 4 and 5, is also reflected in Fig. 6: downstream of the vortex breakdown the vorticity is less concentrated, and far less coherent. The position of the vortex core upstream of the breakdown is not completely fixed in time: slight departures from the averaged position have been identified in the LES. Since the velocity gradients in the vortex core are high, these small departures have significant impact on the velocity fluctuation levels near the vortex core (as will be discussed in a subsequent section). 


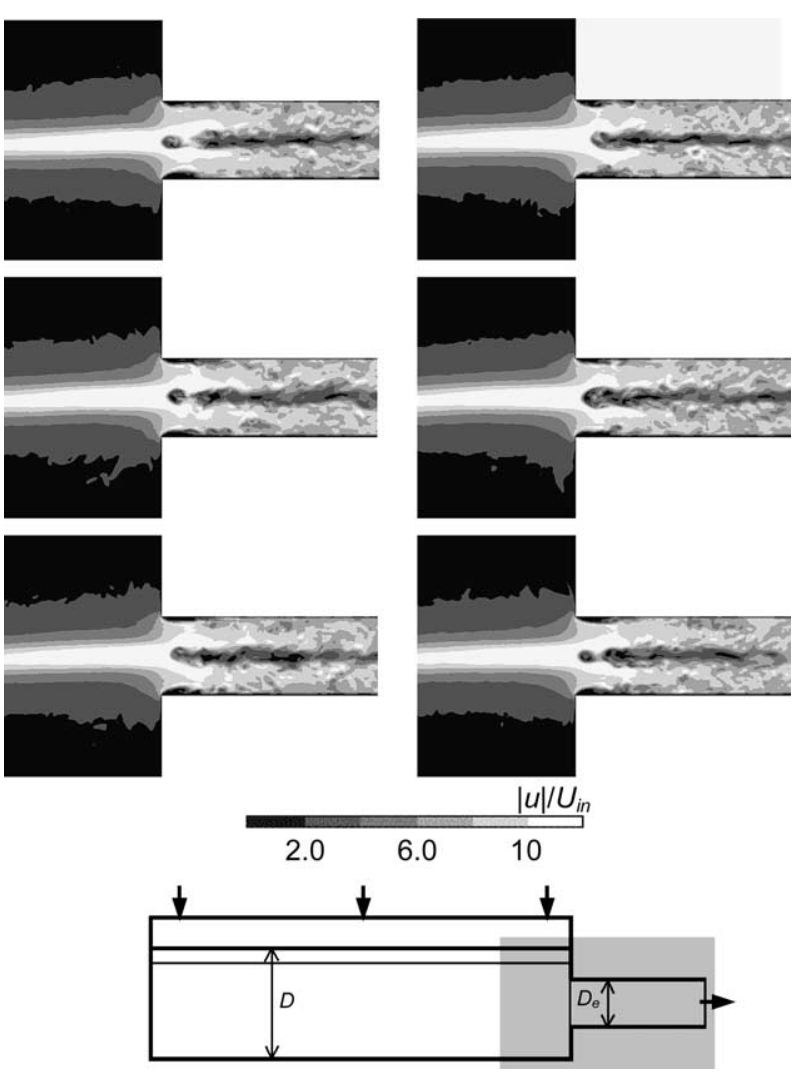

Fig. 5. LES time-sequence of vortex breakdown for $D_{\mathrm{e}} / D=0.33$ at $R e=1900$ (Case \#7). The gray-scale indicates the absolute value of the velocity. The time interval between the successive images is $\Delta t=0.12 D / U_{\mathrm{in}}$. The time runs from top to bottom, and then from left to right. The field of view has been indicated in the schematic.

\subsection{Average velocity profiles}

A quantitative assessment of the simulations is based on the average velocity profiles measured by Escudier et al. [11]. From their flow visualizations (see e.g. Fig. 3), it was clear that in general the vortex core did not coincide with the geometrical center of the swirl tube. For this reason, they took care of traversing the measurement volume through the vortex core (defined as the position with zero, time-average transverse velocity) in their LDA experiments. The latter procedure has been mimicked in the simulations by determining the point in a cross-section of the vortex tube with zero, time-average $y$ - and $z$-velocity. The LES profiles that are presented in this article are profiles along the $y$-axis, and go through the zero-velocity point. In the experiment, the line along which the profile was taken varied with the vortex core position (in fact the angle of the experimental profile was set in such a way as to traverse the vortex core), and was not reported in [11]. This introduces a slight uncertainty in the comparison of experiment and simulation.

First, the Cases \#1, 2, and 4-7 will be discussed. These are the cases with the standard Smagorinsky subgrid-scale model, at a Reynolds number of approximately 2000, on two grids (viz. with 


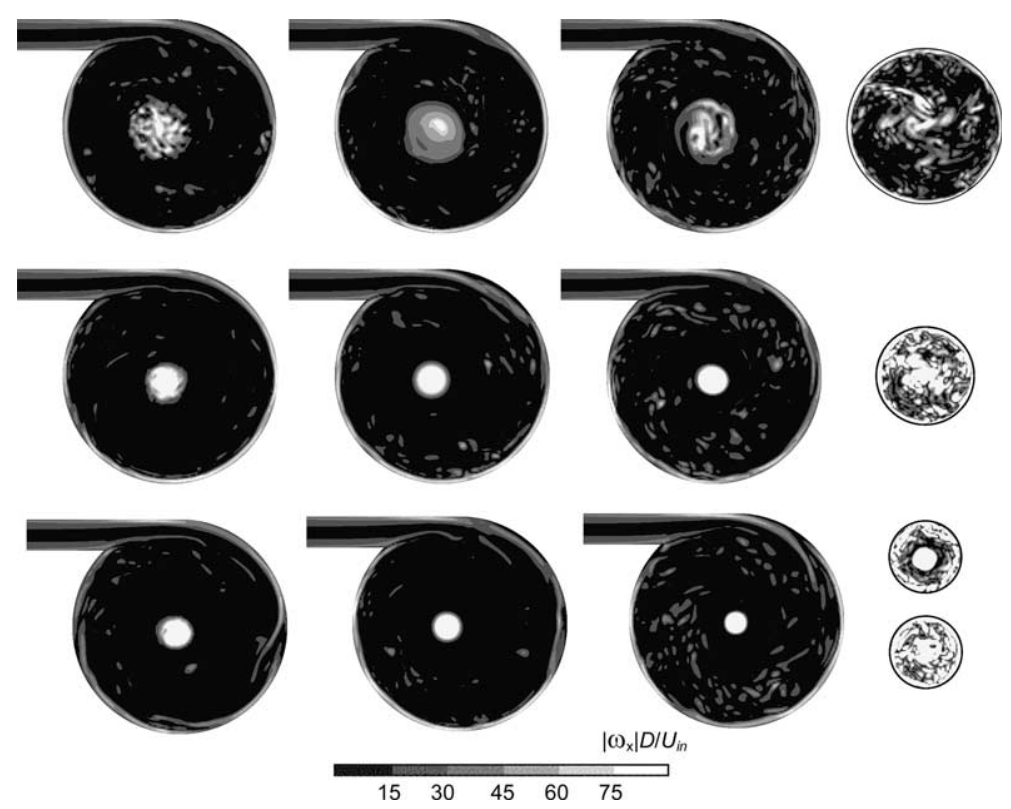

Fig. 6. Cross-sections of the flow in terms of single realizations of the absolute value of the axial vorticity. Top row: $D_{\mathrm{e}} /$ $D=0.73$ (Case \#2); middle row: $D_{\mathrm{e}} / D=0.45$ (Case \#5); bottom row: $D_{\mathrm{e}} / D=0.33$ (Case \#7). From left to right: $x /$ $D=0.15,2.25$, and 3.61. The cross-sections in the exit tube were at $x / D=4.33$ for Case \#2 and 5 ; for Case \#7 one crosssection is upstream the vortex breakdown (at $x / D=3.91$ ) and one is downstream (at $x / D=4.70$ ).

$\Delta=D / 82$ and $\Delta=D / 132$ ). The results have been summarized in Figs. 7 (time-average tangential velocity) and 8 (time-average axial velocity). The radial profiles of the swirl component have a shape similar to Burgers' solution [29]. The latter was based on the assumption of a uniform axial velocity distribution. The actual axial velocity profiles are, however, far from uniform (see Fig. 8).

The geometrical ratio $D_{\mathrm{e}} / D$ has strong impact on the tangential velocity levels: the maximum tangential velocity increases significantly, and the diameter of the vortex core (as a measure for the latter we take the distance between the two extremes in the profile) reduces if $D_{\mathrm{e}} / D$ is reduced. As a consequence, velocity gradients at $D_{\mathrm{e}} / D=0.33$ are much higher than at $D_{\mathrm{e}} / D=0.73$. The experimental tangential velocity profiles only show a weak dependence on the axial position; towards the exit pipe the maximum velocity slightly increases. This trend is strongest for $D_{\mathrm{e}} /$ $D=0.33$. The quality of the LES results appears to be directly related to the gradients in the average flow field. The agreement between simulation and experiment is good for $D_{\mathrm{e}} / D=0.73$, and for $D_{\mathrm{e}} / D=0.45$. For these cases, the levels of maximum tangential velocity, and the vortex core diameter correspond with the experimental data. Furthermore it is reassuring to see that the agreement gets better for higher spatial resolution. For the cases with $D_{\mathrm{e}} / D=0.33$, spatial resolution effects are very pronounced. In this case, the LES profiles are less sharp than the experimental ones, leading to an underestimation of the maximum velocity levels. It is anticipated that the Smagorinsky model is too dissipative here. This will be further discussed when results of simulations with different SGS models are compared.

Also the shape of the radial profiles of the axial velocity component (Fig. 8) strongly depends on the ratio $D_{\mathrm{e}} / D$. Furthermore, there now is a strong dependency on the axial position in the 

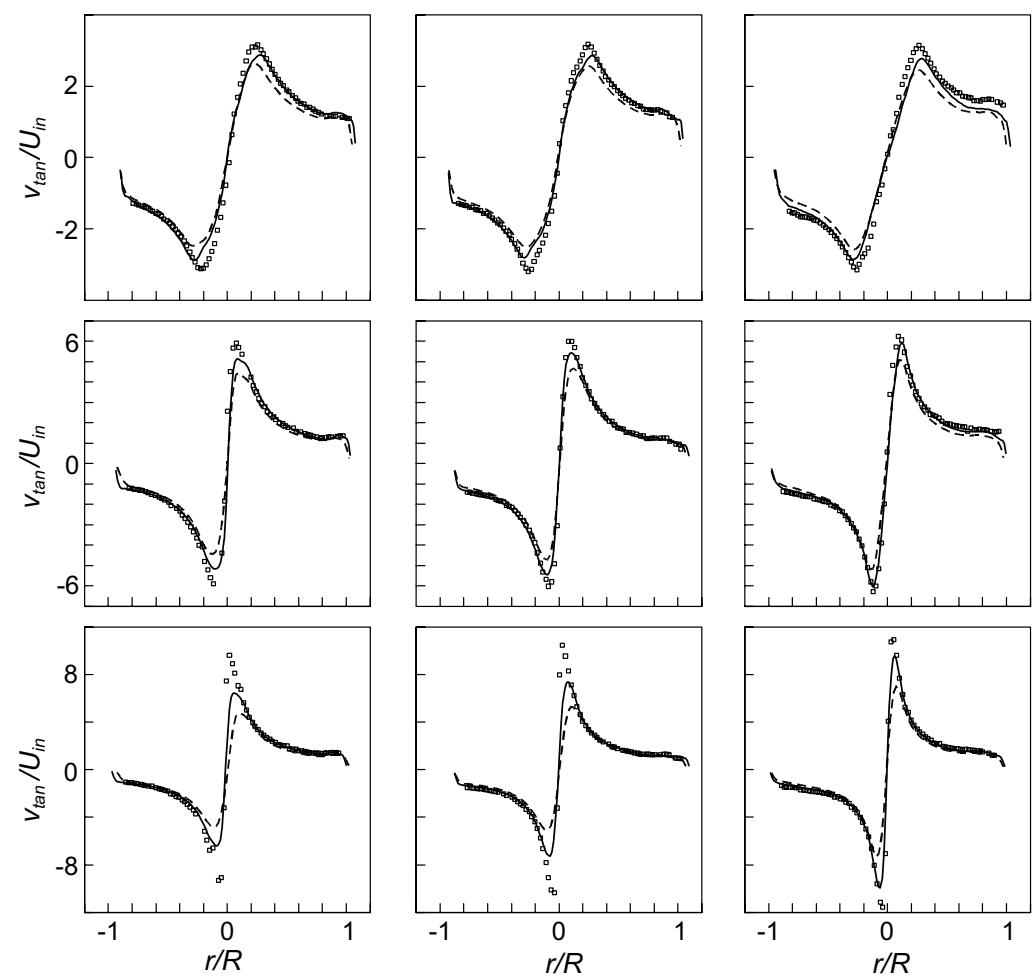

Fig. 7. Tangential velocity profiles. From top to bottom: $D_{\mathrm{e}} / D=0.73, D_{\mathrm{e}} / D=0.45$, and $D_{\mathrm{e}} / D=0.33$. From left to right: $x / D=0.15,2.15$, and 3.61. The symbols denote experimental data [11]. The dashed line is the LES result with $\Delta=D / 82$, the solid line with $\Delta=D / 132 . R=D / 2$.

vortex tube as well. The width of the axial velocity profiles appears to be related to the diameter of the vortex core and since the latter was predicted well, the width of the axial profiles is well represented by the LES. We consider the pressure field responsible for this tight coupling between the axial and tangential velocity. The radial pressure distribution induced by the swirl component, and its (minor) axial variation strongly influences the axial velocity. The profiles at $D_{\mathrm{e}} /$ $D=0.73$, and $D_{\mathrm{e}} / D=0.45$ show an axial velocity deficit near the axis, whereas at $D_{\mathrm{e}} / D=0.33$ the profiles show a single, and sharp peak. In the first two profiles at $D_{\mathrm{e}} / D=0.73$, the reverse flow near the center is not captured by the LES. Strong sensitivity of the LES with respect to the spatial resolution is apparent already at $D_{\mathrm{e}} / D=0.45$. The lower resolution simulation underestimates, or even misses the velocity deficit in the very core of the flow, whereas the higher resolution is capable of representing it to a fair extent. The peak levels at $D_{\mathrm{e}} / D=0.33$ are captured poorly by the LES. For $x / D=0.15,2.15$ the axial velocity peak-level is underestimated, whereas for $x / D=3.61$ it is overestimated (it should be noted, however, that for the sharp profiles encountered at $x /$ $D=3.61$ also the spatial resolution of the LDA comes into play).

By comparing Case \#2 and \#3, we assessed the effect of the Reynolds number on the average flow (Case \#2 has $R e=2100$, \#3 has 4600). It was concluded from the profiles (not shown) that the effect is very limited, which is in accordance with experimental observations reported in [11]. 

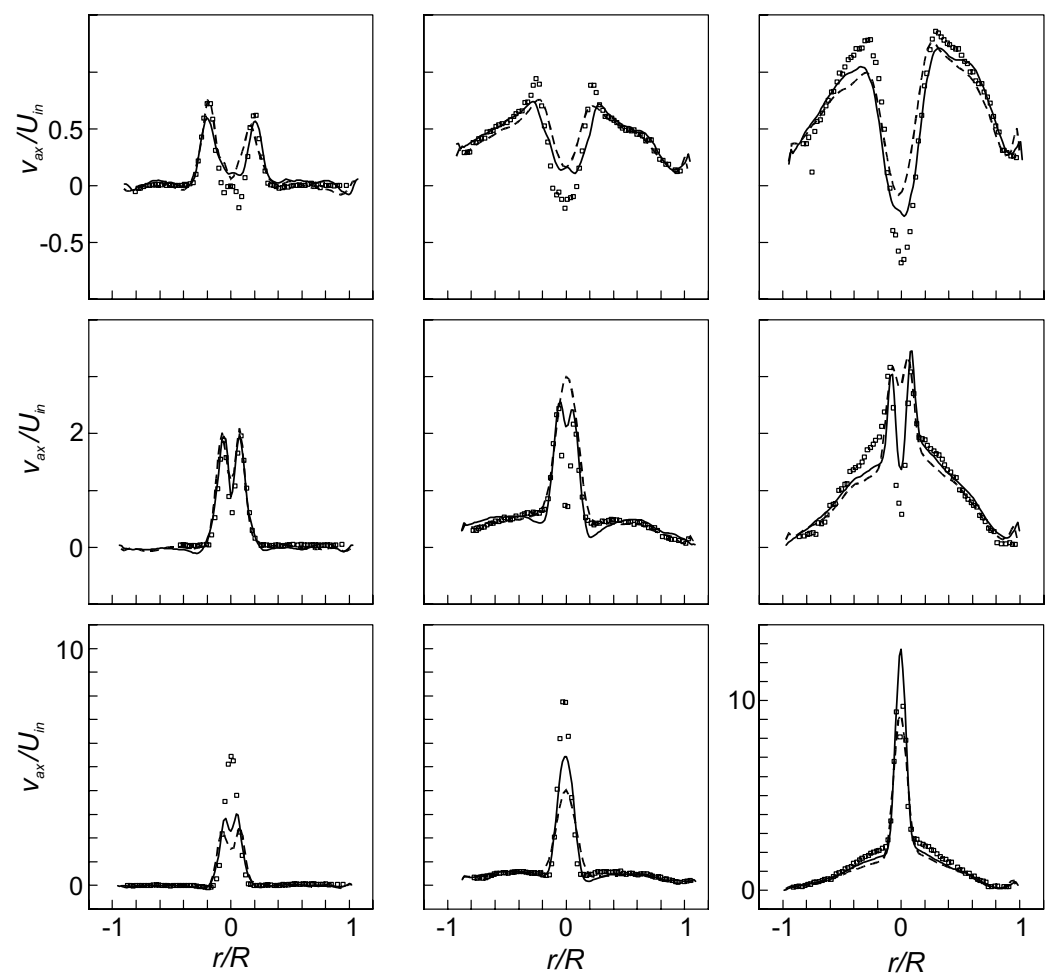

Fig. 8. Axial velocity profiles. From top to bottom: $D_{\mathrm{e}} / D=0.73, D_{\mathrm{e}} / D=0.45$, and $D_{\mathrm{e}} / D=0.33$. From left to right: $x /$ $D=0.15,2.15$, and 3.61. The symbols denote experimental data [11]. The dashed line is the LES result with $\Delta=D / 82$, the solid line with $\Delta=D / 132$.

From now on, we focus on the cases with $D_{\mathrm{e}} / D=0.33$ (Cases \#6-10), since here the most pronounced grid effects were observed (see Figs. 7 and 8). In the first place, the grid was refined further (in Case \#10). In the second place, various SGS models have been applied. Further refining the grid has the obvious effects: higher gradients can be captured, and the simulated profiles tend more to the experimental ones (Fig. 9). It has to be noted here that the computational expense is quite big. The total number of grid nodes in the simulation with the finest grid amounts to approximately 50 million. It therefore is worthwhile to look into other methods to improve the quality of the flow field predictions. This is the motivation to test the performance of other SGS models applied to the flow system at hand.

The profiles in Fig. 10 show the results with the three SGS models applied to the grid with $\Delta=D / 132$. The results obtained with the model due to Voke [15] are very similar to the ones of the standard Smagorinsky model. Only minor differences (that hardly exceed the uncertainties introduced by the finite averaging time) can be observed. The MSM [16] proves to be a significant improvement compared to both the Smagorinsky model, and Voke's adaptation to it: The peak levels of the tangential velocity are now in good agreement with the experimental ones; the MSM simulation performs even better than the (Smagorinsky) simulation on the finest grid. 

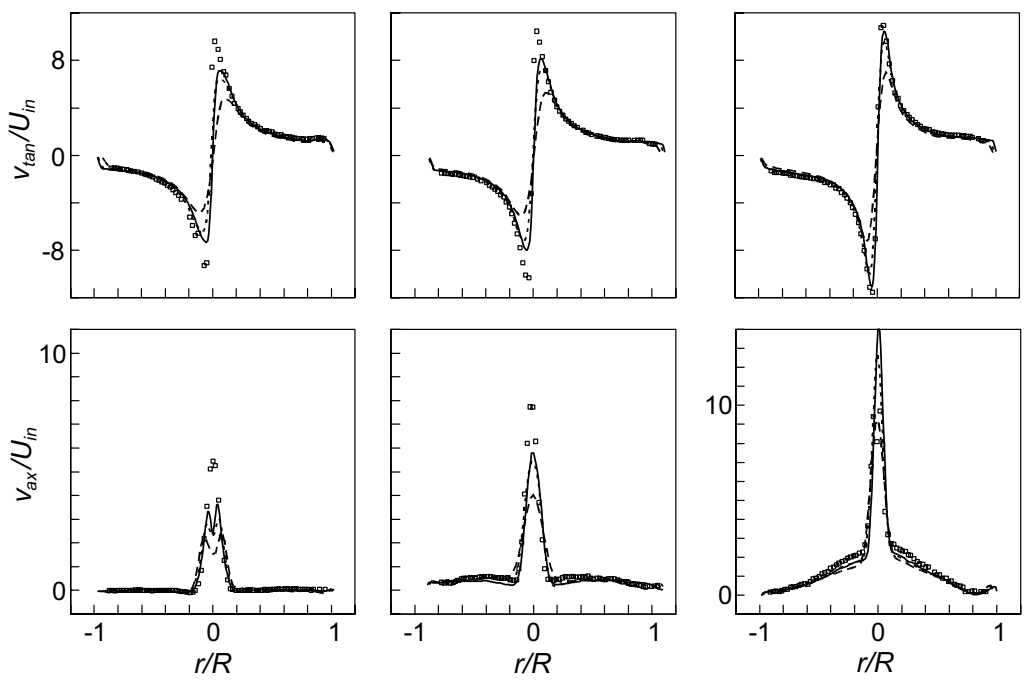

Fig. 9. Average velocity profiles for $D_{\mathrm{e}} / D=0.33$. Top: tangential velocity; bottom: axial velocity. From left to right: $x /$ $D=0.15,2.15$, and 3.61. The symbols denote experimental data [11]. The long-dashed line is the LES result with $\Delta=D /$ 82; the short-dashed line with $\Delta=D / 132$; and the solid line with $\Delta=D / 184$. In the LES the Smagorinsky model was applied.

The differences and similarities between the results obtained with the various SGS models are reflected in the eddy-viscosity profiles in Fig. 10. The viscosities of the Voke model are close to the Smagorinsky values. Only outside the vortex core the adaptation due to Voke reduces the viscosity. The MSM provides low eddy-viscosities throughout the flow. The values are typically smaller by a factor of two compared to the two other models.

\subsection{Velocity fluctuation levels}

Escudier et al. [11] did not report experimental data with respect to velocity fluctuations. Therefore, the LES profiles of the RMS values of the three velocity components displayed in Fig. 11 could not be assessed with measured data. The very pronounced peak in the vortex core is due to small fluctuations of the position of the vortex core. We have kept track of the vortex core position during the simulations. The fluctuations of the vortex core position about its mean have RMS values of the $y$ and $z$ coordinate of approximately $4 \times 10^{-3} \mathrm{D}$ (this value applies to $y$ as well as $z$, the fluctuations were almost fully isotropic). Although the positional fluctuations are small, the high velocity gradients induce high velocity fluctuation levels in the vortex core. Gradients of the tangential velocity in the vortex core for the cases with $D_{\mathrm{e}} / D=0.33$ are of the order of $220 U_{\text {in }} / D$ (see e.g. Fig. 7). Velocity fluctuations levels due to positional fluctuations of the vortex core can then be estimated to have RMS values of the order of $0.9 U_{\text {in }}$, which is slightly lower (but at least of the same order of magnitude) as the peak levels observed in Fig. 11.

In the outer flow, the tangential fluctuations are less strong than axial and radial fluctuations. This is probably due to the dynamics of the Taylor-Görtler vortices in the outer flow. Their structure mainly contributes to radial and axial RMS values. Finally, the shear layer along the outer 

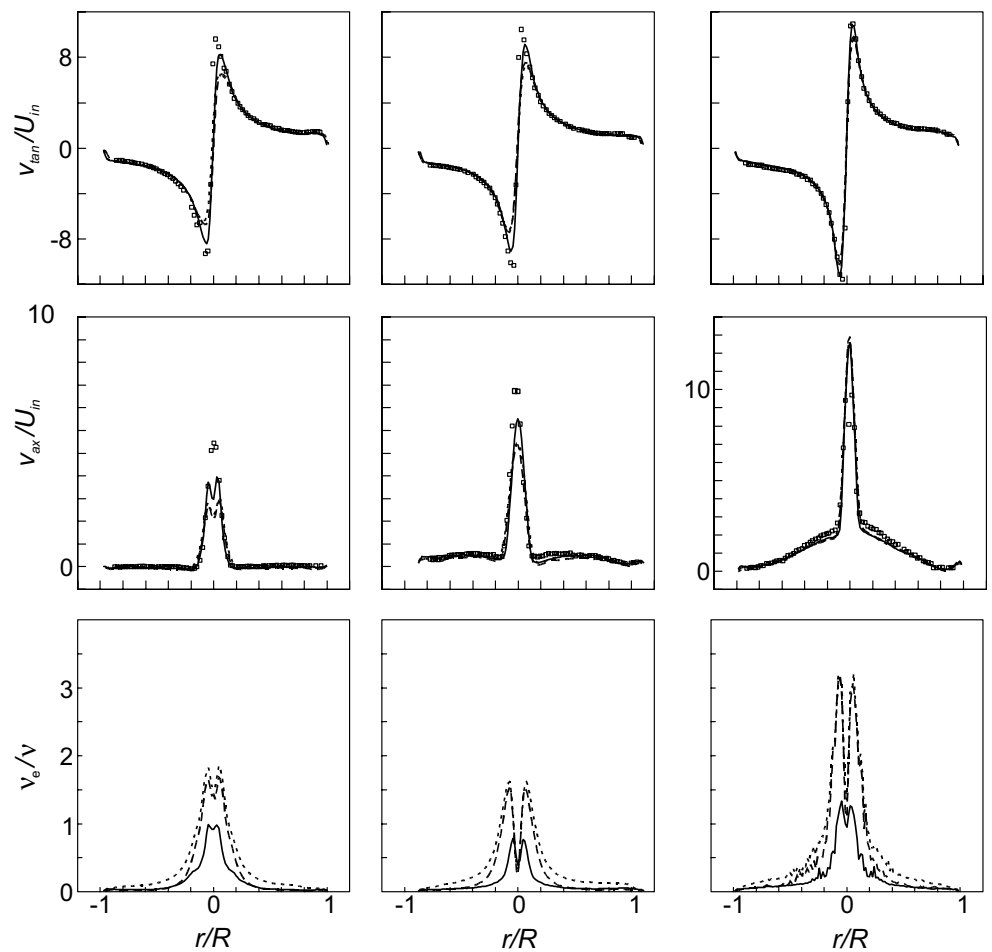

Fig. 10. Average velocity and eddy-viscosity profiles for $D_{\mathrm{e}} / D=0.33$ with a $\Delta=D / 132$ resolution. Top: tangential velocity; middle: axial velocity; bottom: eddy viscosity. From left to right: $x / D=0.15,2.15$, and 3.61 . The symbols denote experimental data [11]. The short-dashed line is the LES result with the Smagorinsky model, the long-dashed line with the model due to Voke, and the solid line with the mixed-scale model.

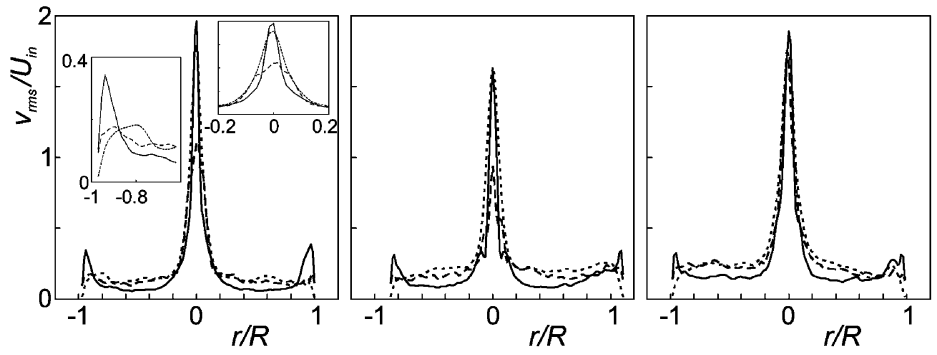

Fig. 11. Radial profiles of the RMS velocity for $D_{\mathrm{e}} / D=0.33$ with a $\Delta=D / 132$ resolution, and a Smagorinsky SGS model. From left to right: $x / D=0.15,2.15$, and 3.61. The short-dashed line is the radial velocity component, the longdashed line the axial component, and the solid line is the tangential component. The insets in the left figure are enlargements of the wall region, and the of the peaks in the center (the vertical scale of the latter runs from 0 to 2 ).

wall induces relatively high levels of tangential velocity fluctuations. Qualitatively, the Reynolds normal stress profiles are in accordance with an experimental study by Kitoh [30]: elevated stress levels in the center region, and close to the wall. 


\section{Conclusions}

The flow in a swirl tube that was experimentally studied more than 20 years ago [11] has been revisited in the present LES study. The numerical simulations employed the lattice-Boltzmann methodology to solve the filtered Navier-Stokes equations. In a qualitative sense, the LES represented the experimentally observed flow features well: The LES showed evidence for laminarization of the vortex core, just as observed in laser sheet visualizations; Taylor-Görtler vortices were seen in the experiment as well as in the LES; and bubble-type vortex breakdown was observed at similar locations as in the experiments. Besides e.g. the work by Spall and co-workers [31,32], this is one of the first reports of three-dimensional simulation of turbulent vortex breakdown in conjunction with experimental validation.

LDA data allowed for a quantitative assessment of the numerical results. The entire flow field is strongly influenced by geometrical changes in the outlet geometry. The swirl velocity more than linearly increases with the ratio of swirl tube diameter over exit pipe diameter $D /$

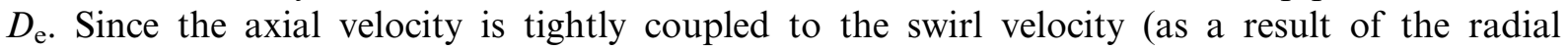
and axial pressure profiles induced by the swirl), its profiles are also a pronounced function of the exit pipe diameter. The LDA data measured with various exit pipe diameters gave us the opportunity to explore the limitations of our simulation procedure. The simulations with the narrower exit pipes have very high velocity gradients, which demand highly resolved simulations, and appropriate (i.e. not too dissipative) subgrid-scale models. The performance of three SGS models (viz. the standard Smagorinsky model [14], an adaptation of the Smagorinsky model due to Voke [15], and a mixed-scale-model due to Sagaut et al. [16]) was compared in terms of the time-averaged velocity profiles. The MSM performed best due to its low damping. Hardly any reduction of the eddy-viscosity was observed due to Voke's model, and as a result the model does not perform better than the Smagorinsky model for the present flow system.

The velocity fluctuation levels of the turbulent flow are highest in the core region. These fluctuations are apparently induced by the high velocity gradients in the core, and the very weak motion of the core, albeit that this combined effect cannot fully account for the peak values. In the outer region, the radial and axial velocity fluctuations are significantly higher than the tangential fluctuations.

A critical issue of the simulations is the treatment of solid walls. In the first place since the lattice-Boltzmann method employs a uniform cubic grid, in the second place since the SGS models used need to be supplemented with wall damping functions to explicitly bring the eddy-viscosity to zero at the solid surfaces. The use of stair-step surfaces has been successfully avoided by making use of an immersed boundary method. Van Driest damping functions were applied at all solid surfaces. This relatively simple approach led to good predictions of a complicated flow.

\section{Acknowledgement}

I am grateful to professor Marcel Escudier for making available his experimental data. 


\section{References}

[1] Gupta AK, Lilley DG, Syred N. Swirl flows. Tunbridge Wells: Abacus Press; 1984.

[2] Escudier M. Vortex breakdown, observations and explanations. Progr Aerospace Sci 1988;25:189-229.

[3] Sarpkaya T. Turbulent vortex breakdown. Phys Fluids 1995;7:2301-3.

[4] Alekseenko SV, Kuibin PA, Okulov VL, Shtork SI. Helical vortices in swirl flow. J Fluid Mech 1999;382:195-243.

[5] Goldshtik M, Hussain F. The nature of inviscid vortex breakdown. Phys Fluids 1997;9:263-5.

[6] Derksen J, Van den Akker HEA. Simulation of vortex core precession in a reverse-flow cyclone. AIChE J 2000;46:1317-31.

[7] Derksen J. Separation performance predictions of a Sairmand high-efficiency cyclone. AIChE J 2003;49:1359-71.

[8] Wunenburger R, Andreotti B, Petitjeans P. Influence of precession on velocity measurements in a strong laboratory vortex. Exp Fluids 1999;27:181-8.

[9] Yazdabadi PA, Griffiths AJ, Syred N. Characterization of the PVC phenomena in the exhaust of a cyclone dust separator. Exp Fluids 1994;17:84-95.

[10] Jakirlic S, Hanjalic K, Tropea C. Modeling Rotating and Swirling Turbulent Flows: A Perpetual Challenge. AIAA J 2002;40:1984-96.

[11] Escudier MP, Bornstein J, Zehnder N. Observations and LDA measurements of confined turbulent vortex flow. J Fluid Mech 1980;98:49-63.

[12] Pierce CD, Moin P. Method for generating equilibrium swirling inflow conditions. AIAA J 1998;36:1325-7.

[13] Escudier MP, Bornstein J, Maxworthy T. The dynamics of confined vortices. Proc R Soc London 1982;A382:33560.

[14] Smagorinsky J. General circulation experiments with the primitive equations: part I, the basic experiment. Monthly Weather Rev 1963;91:99-164.

[15] Voke PR. Subgrid-scale modelling at low mesh Reynolds number. Theor Comput Fluid Dyn 1996;8:131-43.

[16] Sagaut P, Comte P, Ducros F. Filtered subgrid-scale models. Phys Fluids 2000;12:233-6.

[17] Hinze JO. Turbulence. New York: McGraw-Hill; 1959.

[18] Chen S, Doolen GD. Lattice Boltzmann method for fluid flows. Annu Rev Fluid Mech 1998;30:329-64.

[19] Somers JA. Direct simulation of fluid flow with cellular automata and the lattice-Boltzmann equation. Appl Sci Res 1993;51:127-33.

[20] Eggels JGM, Somers JA. Numerical simulation of free convective flow using the lattice-Boltzmann scheme. Int J Heat Fluid Flow 1995;16:357-64.

[21] Eggels JGM. Direct and large-eddy simulations of turbulent fluid flow using the lattice-Boltzmann scheme. Int J Heat Fluid Flow 1996;17:307-23.

[22] Derksen JJ, Van den Akker HEA. Large eddy simulations on the flow driven by a Rushton turbine. AIChE J 1999;45:209-21.

[23] Goldstein D, Handler R, Sirovich L. Modeling a no-slip flow boundary with an external force field. J Comput Phys 1993;105:354-66.

[24] Balaras E. Modeling complex boundaries using an external force field on fixed Cartesian grids in large-eddy simulations. Comput Fluids 2004;33:375-404.

[25] Verzicco R, Mohod-Yusof J, Orlandi P, Haworth D. Large-eddy-simulation in complex geometric configurations using boundary body forces. AIAA J 2000;38:427-33.

[26] Derksen JJ. Numerical simulation of solids suspension in a stirred tank. AIChE J 2003;49:2700-14.

[27] Ten Cate A, Nieuwstad CH, Derksen JJ, Van den Akker HEA. Particle image velocimetry experiments and latticeBoltzmann simulations on a single sphere settling under gravity. Phys Fluids 2002;14:4012-25.

[28] Faler JH, Leibovich S. Disrupted states of vortex flow and vortex breakdown. Phys Fluids 1977;20:1385-400.

[29] Burgers JM. A mathematical model illustrating the theory of turbulence. Adv Appl Mech 1948;1:171-98.

[30] Kitoh O. Experimental study of turbulent swirling flow in a straight pipe. J Fluid Mech 1991;25:445-79.

[31] Snyder DE, Spall RE. Numerical simulation of bubble-type vortex breakdown within a tube-and-vane apparatus. Phys Fluids 2000;12:603-8.

[32] Spall RE, Ashby BM. A numerical study of vortex breakdown in turbulent swirling flows. J Fluids Eng 2000;122:179-83. 